\title{
Electron Donation from Cu Atoms to Al Oxide Clusters Upon Mixing Revealed by Thermal Desorption Spectrometry
}

Fumitaka Mafuné*, Yufei Zhang, Xinan Liu, Haohao Wang and Satoshi Kudoh

Department of Basic Science, School of Arts and Sciences,

The University of Tokyo, Komaba, Meguro, Tokyo 153-8902, JAPAN

Email: mafune@cluster.c.u-tokyo.ac.jp

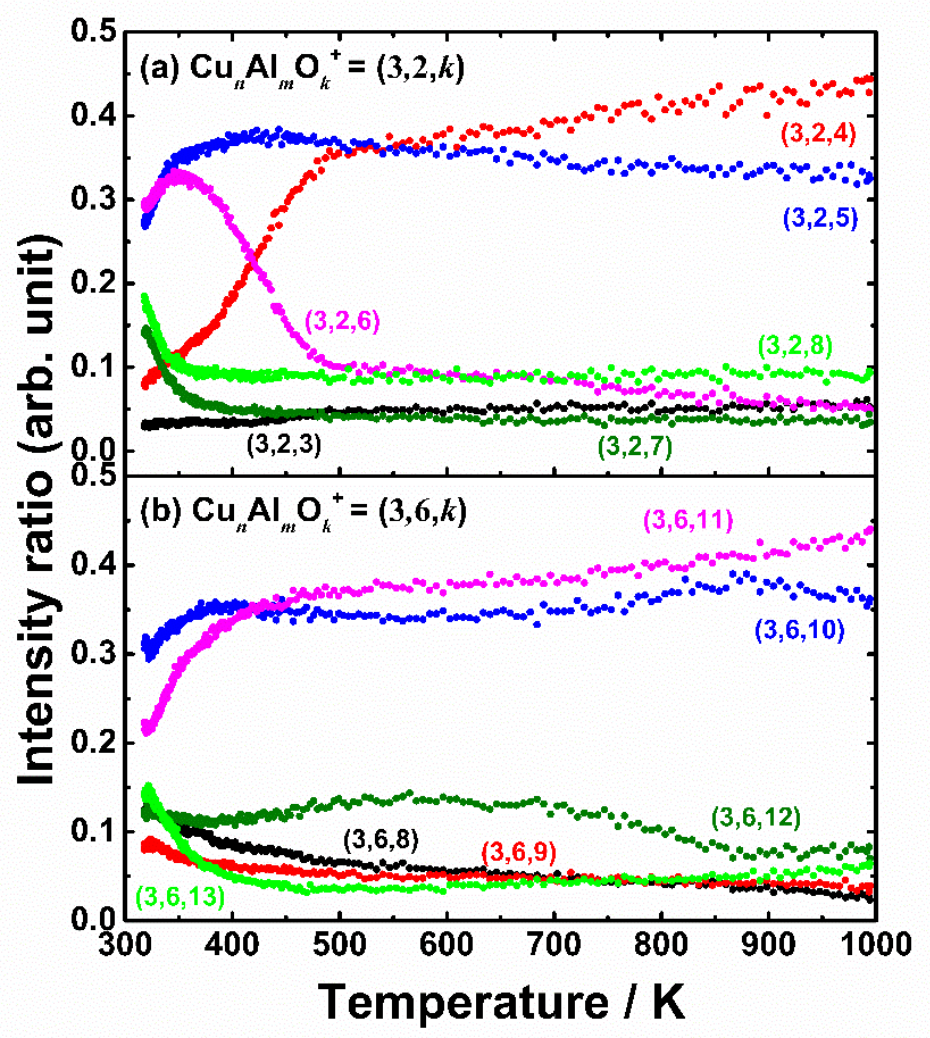

Figure S1 TDS plots of $\mathrm{Cu}_{3} \mathrm{Al}_{2} \mathrm{O}_{k}{ }^{+}$and $\mathrm{Cu}_{3} \mathrm{Al}_{6} \mathrm{O}_{k}{ }^{+}$. 


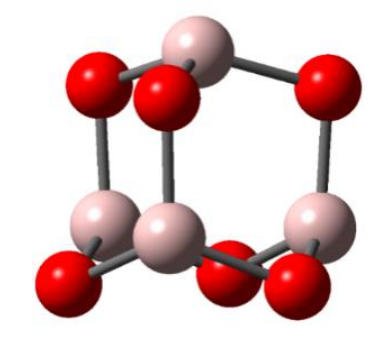

Figure S2 Geometrical structure of the most stable neutral $\mathrm{Al}_{4} \mathrm{O}_{6}$ cluster. Natural charges of all the $\mathrm{Al}$ atoms and all the $\mathrm{O}$ atoms are $+2.11 \mathrm{e}$ and $-1.40 \mathrm{e}$, respectively, according to the NBO analysis. Pink and red balls represent $\mathrm{Al}$ and $\mathrm{O}$ atoms, respectively.

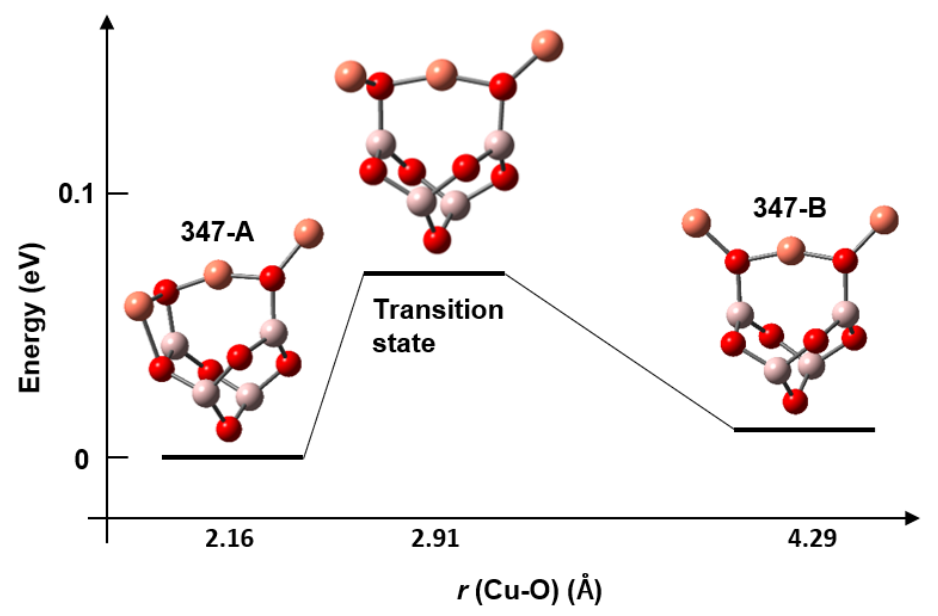

Figure S3 Energy diagram for isomerization between 347-A and 347-B. The bond length, $r(\mathrm{Cu}-$ $\mathrm{O})$, represent the distance between $\mathrm{Cu}(12)$ and $\mathrm{O}(8)$ labeled in Figure 6. 
Table S1 Wiberg bond index of $\mathrm{Cu}_{3} \mathrm{Al}_{4} \mathrm{O}_{7}^{+}$obtained by DFT calculation.

347-A

\begin{tabular}{|c|c|c|c|c|c|c|c|c|c|c|c|c|c|c|c|}
\hline & & Al & Al & Al & Al & O & O & O & O & O & O & 0 & $\mathrm{Cu}$ & $\mathrm{Cu}$ & $\mathrm{Cu}$ \\
\hline & & 1 & 2 & 3 & 4 & 5 & 6 & 7 & 8 & 9 & 10 & 11 & 12 & 13 & 14 \\
\hline Al & 1 & 0 & 0.0164 & 0.0274 & 0.0174 & 0.518 & 0.0057 & 0.0021 & 0.4161 & 0.4769 & 0.0059 & 0.0079 & 0.0071 & 0.0023 & 0.0005 \\
\hline Al & 2 & 0.0164 & 0 & 0.0185 & 0.005 & 0.0047 & 0.0032 & 0.0016 & 0.4049 & 0.0041 & 0.5419 & 0.5265 & 0.0266 & 0.0583 & 0.0007 \\
\hline Al & 3 & 0.0274 & 0.0185 & 0 & 0.0192 & 0.4879 & 0.4771 & 0.0023 & 0.0034 & 0.0066 & 0.0024 & 0.4524 & 0.0007 & 0.0017 & 0.0003 \\
\hline Al & 4 & 0.0174 & 0.005 & 0.0192 & 0 & 0.0035 & 0.4999 & 0.4478 & 0.0021 & 0.479 & 0.0049 & 0.0033 & 0.0014 & 0.0635 & 0.014 \\
\hline O & 5 & 0.518 & 0.0047 & 0.4879 & 0.0035 & 0 & 0.0192 & 0.0007 & 0.0165 & 0.0205 & 0.0011 & 0.0202 & 0.0039 & 0.0011 & 0.0002 \\
\hline O & 6 & 0.0057 & 0.0032 & 0.4771 & 0.4999 & 0.0192 & 0 & 0.0132 & 0.0012 & 0.0229 & 0.0012 & 0.016 & 0.0006 & 0.0093 & 0.002 \\
\hline O & 7 & 0.0021 & 0.0016 & 0.0023 & 0.4478 & 0.0007 & 0.0132 & 0 & 0.0004 & 0.0122 & 0.0156 & 0.0008 & 0.0025 & 0.2338 & 0.1785 \\
\hline O & 8 & 0.4161 & 0.4049 & 0.0034 & 0.0021 & 0.0165 & 0.0012 & 0.0004 & 0 & 0.0142 & 0.0183 & 0.0186 & 0.0851 & 0.0078 & 0.0001 \\
\hline O & 9 & 0.4769 & 0.0041 & 0.0066 & 0.479 & 0.0205 & 0.0229 & 0.0122 & 0.0142 & 0 & 0.0023 & 0.0027 & 0.003 & 0.0142 & 0.0042 \\
\hline O & 10 & 0.0059 & 0.5419 & 0.0024 & 0.0049 & 0.0011 & 0.0012 & 0.0156 & 0.0183 & 0.0023 & 0 & 0.0168 & 0.1538 & 0.307 & 0.0051 \\
\hline O & 11 & 0.0079 & 0.5265 & 0.4524 & 0.0033 & 0.0202 & 0.016 & 0.0008 & 0.0186 & 0.0027 & 0.0168 & 0 & 0.0051 & 0.0091 & 0.0002 \\
\hline $\mathrm{Cu}$ & 12 & 0.0071 & 0.0266 & 0.0007 & 0.0014 & 0.0039 & 0.0006 & 0.0025 & 0.0851 & 0.003 & 0.1538 & 0.0051 & 0 & 0.0611 & 0.0011 \\
\hline $\mathrm{Cu}$ & 13 & 0.0023 & 0.0583 & 0.0017 & 0.0635 & 0.0011 & 0.0093 & 0.2338 & 0.0078 & 0.0142 & 0.307 & 0.0091 & 0.0611 & 0 & 0.0584 \\
\hline $\mathrm{Cu}$ & 14 & 0.0005 & 0.0007 & 0.0003 & 0.014 & 0.0002 & 0.002 & 0.1785 & .0001 & 0042 & 0051 & .0002 & .0011 & 0.05 & \\
\hline
\end{tabular}

347-B

\begin{tabular}{|c|c|c|c|c|c|c|c|c|c|c|c|c|c|c|c|}
\hline & & Al & Al & Al & Al & o & o & o & o & o & o & o & $\mathrm{Cu}$ & $\mathrm{Cu}$ & $\mathrm{Cu}$ \\
\hline & & 1 & 2 & 3 & 4 & 5 & 6 & 7 & 8 & 9 & 10 & 11 & 12 & 13 & 14 \\
\hline Al & 1 & 0 & 0.0285 & 0.0206 & 0.018 & 0.5024 & 0.0047 & 0.002 & 0.4713 & 0.4625 & 0.0022 & 0.0074 & 0.0004 & 0.0022 & 0.0006 \\
\hline Al & 2 & 0.0285 & 0 & 0.018 & 0.0206 & 0.5024 & 0.4713 & 0.0022 & 0.0047 & 0.0074 & 0.002 & 0.4625 & 0.0006 & 0.0022 & 0.0004 \\
\hline Al & 3 & 0.0206 & 0.018 & 0 & 0.0044 & 0.004 & 0.0028 & 0.0018 & 0.5085 & 0.0041 & 0.4345 & 0.5035 & 0.0147 & 0.0523 & 0.0006 \\
\hline Al & 4 & 0.018 & 0.0206 & 0.0044 & 0 & 0.004 & 0.5085 & 0.4345 & 0.0028 & 0.5035 & 0.0018 & 0.0041 & 0.0006 & 0.0523 & 0.0147 \\
\hline O & 5 & 0.5024 & 0.5024 & 0.004 & 0.004 & 0 & 0.0189 & 0.0006 & 0.0189 & 0.0199 & 0.0006 & 0.0199 & 0.0002 & 0.0015 & 0.0002 \\
\hline $\mathbf{O}$ & 6 & 0.0047 & 0.4713 & 0.0028 & 0.5085 & 0.0189 & 0 & 0.013 & 0.0015 & 0.0241 & 0.0007 & 0.016 & 0.0004 & 0.0099 & 0.0026 \\
\hline O & 7 & 0.002 & 0.0022 & 0.0018 & 0.4345 & 0.0006 & 0.013 & 0 & 0.0007 & 0.0122 & 0.0123 & 0.0011 & 0.0024 & 0.2253 & 0.1871 \\
\hline $\mathbf{O}$ & 8 & 0.4713 & 0.0047 & 0.5085 & 0.0028 & 0.0189 & 0.0015 & 0.0007 & 0 & 0.016 & 0.0129 & 0.0241 & 0.0026 & 0.0099 & 0.0004 \\
\hline o & 9 & 0.4625 & 0.0074 & 0.0041 & 0.5035 & 0.0199 & 0.0241 & 0.0122 & 0.016 & 0 & 0.0011 & 0.003 & 0.0005 & 0.0124 & 0.0053 \\
\hline O & 10 & 0.0022 & 0.002 & 0.4345 & 0.0018 & 0.0006 & 0.0007 & 0.0123 & 0.0129 & 0.0011 & 0 & 0.0122 & 0.1871 & 0.2253 & 0.0024 \\
\hline O & 11 & 0.0074 & 0.4625 & 0.5035 & 0.0041 & 0.0199 & 0.016 & 0.0011 & 0.0241 & 0.003 & 0.0122 & 0 & 0.0053 & 0.0124 & 0.0005 \\
\hline $\mathrm{Cu}$ & 12 & 0.0004 & 0.0006 & 0.0147 & 0.0006 & 0.0002 & 0.0004 & 0.0024 & 0.0026 & 0.0005 & 0.1871 & 0.0053 & 0 & 0.0597 & 0.0011 \\
\hline $\mathrm{Cu}$ & 13 & 0.0022 & 0.0022 & 0.0523 & 0.0523 & 0.0015 & 0.0099 & 0.2253 & 0.0099 & 0.0124 & 0.2253 & 0.0124 & 0.0597 & 0 & 0.0596 \\
\hline $\mathrm{Cu}$ & 14 & 0.0006 & 0.0004 & 0.0006 & 0.0147 & 0.0002 & 0.0026 & 0.1871 & 0.0004 & 0.0053 & 0.0024 & 0.0005 & 0.0011 & 0.0596 & 0 \\
\hline
\end{tabular}

347-C

\begin{tabular}{|c|c|c|c|c|c|c|c|c|c|c|c|c|c|c|c|}
\hline & & Al & Al & Al & Al & O & O & O & O & O & O & O & $\mathrm{Cu}$ & $\mathrm{Cu}$ & $\mathrm{Cu}$ \\
\hline & & 1 & 2 & 3 & 4 & 5 & 6 & 7 & 8 & 9 & 10 & 11 & 12 & 13 & 14 \\
\hline Al & 1 & 0 & 0.0167 & 0.039 & 0.0011 & 0.5261 & 0.0045 & 0.0021 & 0.5155 & 0.3763 & 0.0025 & 0.0126 & 0.0004 & 0.0007 & 0.0124 \\
\hline Al & 2 & 0.0167 & 0 & 0.0005 & 0.0134 & 0.0017 & 0.0028 & 0.0064 & 0.474 & 0.0043 & 0.4659 & 0.4533 & 0.0147 & 0.0537 & 0.0115 \\
\hline Al & 3 & 0.039 & 0.0005 & 0 & 0.021 & 0.5394 & 0.5366 & 0.0029 & 0.0039 & 0.3633 & 0.0002 & 0.0086 & 0 & 0.0006 & 0.0133 \\
\hline Al & 4 & 0.0011 & 0.0134 & 0.021 & 0 & 0.0021 & 0.465 & 0.5256 & 0.0031 & 0.0188 & 0.0015 & 0.4406 & 0.0007 & 0.0294 & 0.0621 \\
\hline O & 5 & 0.5261 & 0.0017 & 0.5394 & 0.0021 & 0 & 0.0151 & 0.0013 & 0.0134 & 0.0362 & 0.0003 & 0.0035 & 0.0001 & 0.0008 & 0.0127 \\
\hline o & 6 & 0.0045 & 0.0028 & 0.5366 & 0.465 & 0.0151 & 0 & 0.0148 & 0.0008 & 0.0172 & 0.0005 & 0.0135 & 0.0003 & 0.0041 & 0.0163 \\
\hline O & 7 & 0.0021 & 0.0064 & 0.0029 & 0.5256 & 0.0013 & 0.0148 & 0 & 0.0009 & 0.0118 & 0.0108 & 0.0213 & 0.0049 & 0.2767 & 0.2384 \\
\hline o & 8 & 0.5155 & 0.474 & 0.0039 & 0.0031 & 0.0134 & 0.0008 & 0.0009 & 0 & 0.013 & 0.0105 & 0.0152 & 0.0015 & 0.0043 & 0.0052 \\
\hline o & 9 & 0.3763 & 0.0043 & 0.3633 & 0.0188 & 0.0362 & 0.0172 & 0.0118 & 0.013 & 0 & 0.0006 & 0.0056 & 0.0002 & 0.0038 & 0.1722 \\
\hline o & 10 & 0.0025 & 0.4659 & 0.0002 & 0.0015 & 0.0003 & 0.0005 & 0.0108 & 0.0105 & 0.0006 & o & 0.013 & 0.1803 & 0.1946 & 0.0057 \\
\hline O & 11 & 0.0126 & 0.4533 & 0.0086 & 0.4406 & 0.0035 & 0.0135 & 0.0213 & 0.0152 & 0.0056 & 0.013 & 0 & 0.0049 & 0.0263 & 0.0108 \\
\hline Cu & 12 & 0.0004 & 0.0147 & 0 & 0.0007 & 0.0001 & 0.0003 & 0.0049 & 0.0015 & 0.0002 & 0.1803 & 0.0049 & 0 & 0.0467 & 0.0017 \\
\hline $\mathrm{Cu}$ & 13 & 0.0007 & 0.0537 & 0.0006 & 0.0294 & 0.0008 & 0.0041 & 0.2767 & 0.0043 & 0.0038 & 0.1946 & 0.0263 & 0.0467 & 0 & 0.0948 \\
\hline $\mathbf{u}$ & 14 & 0.0124 & 0.0115 & 0.0133 & 0.0621 & 0.0127 & 0.0163 & 0.2384 & .0052 & 0.1722 & .0057 & 0.0108 & .0017 & .0948 & \\
\hline
\end{tabular}


347-D

\begin{tabular}{|c|c|c|c|c|c|c|c|c|c|c|c|c|c|c|c|}
\hline & & Al & Al & Al & Al & O & o & o & O & O & O & O & $\mathrm{Cu}$ & $\mathrm{Cu}$ & $\mathrm{Cu}$ \\
\hline & & 1 & 2 & 3 & 4 & 5 & 6 & 7 & 8 & 9 & 10 & 11 & 12 & 13 & 14 \\
\hline Al & 1 & 0 & 0.0158 & 0.0263 & 0.0184 & 0.5029 & 0.0041 & 0.0026 & 0.429 & 0.4652 & 0.0065 & 0.0066 & 0.0063 & 0.0019 & 0.0007 \\
\hline Al & 2 & 0.0158 & 0 & 0.0184 & 0.0052 & 0.0045 & 0.0023 & 0.0034 & 0.4129 & 0.0032 & 0.5509 & 0.5066 & 0.0277 & 0.0677 & 0.0018 \\
\hline Al & 3 & 0.0263 & 0.0184 & 0 & 0.0158 & 0.503 & 0.4289 & 0.0065 & 0.0041 & 0.0066 & 0.0026 & 0.4652 & 0.0007 & 0.0019 & 0.0063 \\
\hline Al & 4 & 0.0184 & 0.0052 & 0.0158 & 0 & 0.0045 & 0.4127 & 0.551 & 0.0023 & 0.5065 & 0.0034 & 0.0032 & 0.0018 & 0.0678 & 0.0277 \\
\hline $\mathbf{O}$ & 5 & 0.5029 & 0.0045 & 0.503 & 0.0045 & 0 & 0.017 & 0.001 & 0.0171 & 0.0206 & 0.001 & 0.0206 & 0.0033 & 0.0011 & 0.0033 \\
\hline o & 6 & 0.0041 & 0.0023 & 0.4289 & 0.4127 & 0.017 & 0 & 0.0183 & 0.0011 & 0.0188 & 0.0007 & 0.0142 & 0.0003 & 0.0077 & 0.0658 \\
\hline O & 7 & 0.0026 & 0.0034 & 0.0065 & 0.551 & 0.001 & 0.0183 & 0 & 0.0007 & 0.0168 & 0.0202 & 0.0016 & 0.0038 & 0.2989 & 0.1596 \\
\hline $\mathbf{O}$ & 8 & 0.429 & 0.4129 & 0.0041 & 0.0023 & 0.0171 & 0.0011 & 0.0007 & 0 & 0.0142 & 0.0183 & 0.0188 & 0.0657 & 0.0077 & 0.0003 \\
\hline O & 9 & 0.4652 & 0.0032 & 0.0066 & 0.5065 & 0.0206 & 0.0188 & 0.0168 & 0.0142 & 0 & 0.0016 & 0.0023 & 0.0022 & 0.011 & 0.0047 \\
\hline O & 10 & 0.0065 & 0.5509 & 0.0026 & 0.0034 & 0.001 & 0.0007 & 0.0202 & 0.0183 & 0.0016 & 0 & 0.0168 & 0.1596 & 0.2988 & 0.0038 \\
\hline O & 11 & 0.0066 & 0.5066 & 0.4652 & 0.0032 & 0.0206 & 0.0142 & 0.0016 & 0.0188 & 0.0023 & 0.0168 & 0 & 0.0047 & 0.011 & 0.0022 \\
\hline $\mathrm{Cu}$ & 12 & 0.0063 & 0.0277 & 0.0007 & 0.0018 & 0.0033 & 0.0003 & 0.0038 & 0.0657 & 0.0022 & 0.1596 & 0.0047 & 0 & 0.0616 & 0.0013 \\
\hline $\mathrm{Cu}$ & 13 & 0.0019 & 0.0677 & 0.0019 & 0.0678 & 0.0011 & 0.0077 & 0.2989 & 0.0077 & 0.011 & 0.2988 & 0.011 & 0.0616 & 0 & 0.0617 \\
\hline $\mathrm{Cu}$ & 14 & 0.0007 & 0.0018 & 0.0063 & 0.0277 & 0.0033 & 0.0658 & 0.1596 & 0.0003 & 0.0047 & 0.0038 & 0.0022 & 0.0013 & 0.0617 & 0 \\
\hline
\end{tabular}

347-E

\begin{tabular}{|c|c|c|c|c|c|c|c|c|c|c|c|c|c|c|c|}
\hline & & Al & Al & Al & Al & o & o & O & o & o & o & O & $\mathrm{Cu}$ & $\mathrm{Cu}$ & $\mathrm{Cu}$ \\
\hline & & 1 & 2 & 3 & 4 & 5 & 6 & 7 & 8 & 9 & 10 & 11 & 12 & 13 & 14 \\
\hline Al & 1 & 0 & 0.0138 & 0.0281 & 0.0072 & 0.566 & 0.0067 & 0.0015 & 0.5352 & 0.3116 & 0.0024 & 0.0037 & 0.0003 & 0.0014 & 0.0047 \\
\hline Al & 2 & 0.0138 & 0 & 0.0002 & 0.0267 & 0.0028 & 0.0006 & 0.0043 & 0.4518 & 0.0038 & 0.48 & 0.4811 & 0.0148 & 0.0467 & .001 \\
\hline Al & 3 & 0.0281 & 0.0002 & 0 & 0.0069 & 0.4893 & 0.7823 & 0.0014 & 0.0029 & 0.2466 & 0.0001 & 0.0028 & 0.0001 & 0.0015 & 0.065 \\
\hline Al & 4 & 0.0072 & 0.0267 & 0.0069 & 0 & 0.011 & 0.007 & 0.5831 & 0.0047 & 0.3398 & 0.003 & 0.4962 & 0.0009 & 0.0599 & 0.0612 \\
\hline O & 5 & 0.566 & 0.0028 & 0.4893 & 0.011 & 0 & 0.0188 & 0.001 & 0.0134 & 0.0253 & 0.0004 & 0.0014 & 0.0001 & 0.0012 & 0.004 \\
\hline O & 6 & 0.0067 & 0.0006 & 0.7823 & 0.007 & 0.0188 & 0 & 0.0136 & 0.0011 & 0.0149 & 0.0002 & 0.0007 & 0.0003 & 0.0051 & 0.3325 \\
\hline O & 7 & 0.0015 & 0.0043 & 0.0014 & 0.5831 & 0.001 & 0.0136 & 0 & 0.0011 & 0.0132 & 0.0102 & 0.0231 & 0.0037 & 0.2279 & 0.2129 \\
\hline O & 8 & 0.5352 & 0.4518 & 0.0029 & 0.0047 & 0.0134 & 0.0011 & 0.0011 & 0 & 0.0138 & 0.0108 & 0.0186 & 0.0014 & 0.0054 & .0009 \\
\hline O & 9 & 0.3116 & 0.0038 & 0.2466 & 0.3398 & 0.0253 & 0.0149 & 0.0132 & 0.0138 & 0 & 0.0005 & 0.0122 & 0.0001 & 0.0033 & .0117 \\
\hline O & 10 & 0.0024 & 0.48 & 0.000 & 0.003 & 0.0004 & 0.0002 & 0.0102 & 0.0108 & 0.0005 & 0 & 0.0161 & 0.1757 & 0.1734 & .0011 \\
\hline O & 11 & 0.0037 & 0.4811 & 0.002 & 0.4962 & 0.0014 & 0.0007 & 0.0231 & 0.0186 & 0.0122 & 0.0161 & 0 & 0.0056 & 0.0295 & .004 \\
\hline $\mathrm{Cu}$ & 12 & 0.0003 & 0.0148 & 0.0001 & 0.0009 & 0.0001 & 0.0003 & 0.0037 & 0.0014 & 0.0001 & 0.1757 & 0.0056 & 0 & 0.0459 & 0.0013 \\
\hline Cu & 13 & 0.0014 & 0.0467 & 0.0015 & 0.0599 & .0012 & 0.0051 & 0.2279 & 0.0054 & 0.0033 & 0.1734 & 0.0295 & 0.0459 & 0 & 0.040 \\
\hline $\mathrm{Cu}$ & 14 & 0.0047 & 0.0012 & 0.065 & 0.0612 & 0.004 & 0.3325 & .2129 & 0.0009 & 0.0117 & 0.0011 & 0.0045 & 0.0013 & 0.0407 & \\
\hline
\end{tabular}

Table $\mathbf{S 2}$ Wiberg bond index of $\mathrm{Cu}_{3} \mathrm{Al}_{4} \mathrm{O}_{8}{ }^{+}$obtained by DFT calculation.

348-A

\begin{tabular}{|c|c|c|c|c|c|c|c|c|c|c|c|c|c|c|c|c|}
\hline & & Al & Al & AI & Al & o & o & o & o & o & o & o & o & $\mathrm{Cu}$ & $\mathrm{Cu}$ & $\mathrm{Cu}$ \\
\hline & & 1 & 2 & 3 & 4 & 5 & 6 & 7 & 8 & 9 & 10 & 11 & 12 & 13 & 14 & 15 \\
\hline Al & 1 & 0 & 0.0185 & 0.0316 & 0.0303 & 0.5102 & 0.2463 & 0.0026 & 0.0028 & 0.0017 & 0.2477 & 0.5403 & 0.0025 & 0.0041 & 0.0005 & 0.0004 \\
\hline Al & 2 & 0.0185 & 0 & 0.0079 & 0.0216 & 0.0066 & 0.3754 & 0.0052 & 0.424 & 0.4571 & 0.2839 & 0.0058 & 0.0034 & 0.0255 & 0.0061 & 0.013 \\
\hline Al & 3 & 0.0316 & 0.0079 & 0 & 0.0003 & 0.5499 & 0.3441 & 0.5436 & 0.0012 & 0.0065 & 0.0035 & 0.0033 & 0.0002 & 0.0024 & 0.0001 & 0.0177 \\
\hline Al & 4 & 0.0303 & 0.0216 & 0.0003 & 0 & 0.0024 & 0.003 & 0.0003 & 0.2764 & 0.0018 & 0.261 & 0.5123 & 0.536 & 0.0039 & 0.0221 & 0.0004 \\
\hline o & 5 & 0.5102 & 0.0066 & 0.5499 & 0.0024 & 0 & 0.0265 & 0.014 & 0.0007 & 0.0011 & 0.0078 & 0.0127 & 0.0007 & 0.0033 & 0.0005 & 0.003 \\
\hline o & 6 & 0.2463 & 0.3754 & 0.3441 & 0.003 & 0.0265 & 0 & 0.0126 & 0.0072 & 0.0123 & 0.0162 & 0.0097 & 0.0009 & 0.004 & 0.0024 & 0.0041 \\
\hline o & 7 & 0.0026 & 0.0052 & 0.5436 & 0.0003 & 0.014 & 0.0126 & 0 & 0.0006 & 0.0313 & 0.0008 & 0.0009 & 0.0001 & 0.0084 & 0.0001 & 0.4417 \\
\hline o & 8 & 0.0028 & 0.424 & 0.0012 & 0.2764 & 0.0007 & 0.0072 & 0.0006 & 0 & 0.0088 & 0.0176 & 0.0105 & 0.0368 & 0.0048 & 0.2425 & 0.0024 \\
\hline o & 9 & 0.0017 & 0.4571 & 0.0065 & 0.0018 & 0.0011 & 0.0123 & 0.0313 & 0.0088 & 0 & 0.0117 & 0.0013 & 0.0004 & 0.1032 & 0.0007 & 0.3593 \\
\hline o & 10 & 0.2477 & 0.2839 & 0.0035 & 0.261 & 0.0078 & 0.0162 & 0.0008 & 0.0176 & 0.0117 & 0 & 0.0241 & 0.0077 & 0.1063 & 0.0035 & 0.003 \\
\hline o & 11 & 0.5403 & 0.0058 & 0.0033 & 0.5123 & 0.0127 & 0.0097 & 0.0009 & 0.0105 & 0.0013 & 0.0241 & 0 & 0.0118 & 0.0137 & 0.0044 & 0.0013 \\
\hline o & 12 & 0.0025 & 0.0034 & 0.0002 & 0.536 & 0.0007 & 0.0009 & 0.0001 & 0.0368 & 0.0004 & 0.0077 & 0.0118 & 0 & 0.0014 & 0.4435 & 0.0001 \\
\hline $\mathrm{Cu}$ & 13 & 0.0041 & 0.0255 & 0.0024 & 0.0039 & 0.0033 & 0.004 & 0.0084 & 0.0048 & 0.1032 & 0.1063 & 0.0137 & 0.0014 & 0 & 0.0016 & 0.0266 \\
\hline $\mathrm{Cu}$ & 14 & 0.0005 & 0.0061 & 0.0001 & 0.0221 & 0.0005 & 0.0024 & 0.0001 & 0.2425 & 0.0007 & 0.0035 & 0.0044 & 0.4435 & 0.0016 & 0 & 0.0002 \\
\hline $\mathrm{Cu}$ & 15 & 0.0004 & 0.013 & 0.0177 & 0.0004 & 0.003 & 0.0041 & 0.4417 & 0.0024 & 0.3593 & 0.003 & 0.0013 & 0.0001 & 0.0266 & 0.0002 & o \\
\hline
\end{tabular}


348-B

\begin{tabular}{|c|c|c|c|c|c|c|c|c|c|c|c|c|c|c|c|c|}
\hline & & Al & Al & Al & Al & o & o & o & o & o & o & o & o & $\mathrm{Cu}$ & $\mathrm{Cu}$ & $\mathrm{Cu}$ \\
\hline & & 1 & 2 & 3 & 4 & 5 & 6 & 7 & 8 & 9 & 10 & 11 & 12 & 13 & 14 & 15 \\
\hline Al & 1 & 0 & 0.0087 & 0.0006 & 0.0114 & 0.3439 & 0.4947 & 0.0035 & 0.5603 & 0.0026 & 0.0072 & 0.0008 & 0.0019 & 0.0147 & 0.0105 & 0.0003 \\
\hline Al & 2 & 0.0087 & 0 & 0.0159 & 0.0003 & 0.3495 & 0.0022 & 0.0028 & 0.0088 & 0.4947 & 0.5835 & 0.0016 & 0.0002 & 0.0161 & 0.0154 & 0.0003 \\
\hline Al & 3 & 0.0006 & 0.0159 & 0 & 0.0155 & 0.0189 & 0.0028 & 0.5033 & 0.0005 & 0.4865 & 0.0025 & 0.4325 & 0.0044 & 0.0003 & 0.0523 & 0.0112 \\
\hline Al & 4 & 0.0114 & 0.0003 & 0.0155 & 0 & 0.0033 & 0.4681 & 0.4409 & 0.002 & 0.0025 & 0.0003 & 0.0108 & 0.4997 & 0.0002 & 0.0124 & 0.0165 \\
\hline o & 5 & 0.3439 & 0.3495 & 0.0189 & 0.0033 & 0 & 0.012 & 0.0035 & 0.0126 & 0.0158 & 0.0129 & 0.0091 & 0.0007 & 0.0015 & 0.1604 & 0.001 \\
\hline o & 6 & 0.4947 & 0.0022 & 0.0028 & 0.4681 & 0.012 & 0 & 0.0131 & 0.0118 & 0.0006 & 0.0009 & 0.0011 & 0.0106 & 0.0026 & 0.0114 & 0.0024 \\
\hline o & 7 & 0.0035 & 0.0028 & 0.5033 & 0.4409 & 0.0035 & 0.0131 & 0 & 0.0004 & 0.0134 & 0.0004 & 0.0195 & 0.0146 & 0.0001 & 0.0131 & 0.006 \\
\hline o & 8 & 0.5603 & 0.0088 & 0.0005 & 0.002 & 0.0126 & 0.0118 & 0.0004 & 0 & 0.001 & 0.0299 & 0.0004 & 0.0006 & 0.4157 & 0.0065 & 0.0002 \\
\hline o & 9 & 0.0026 & 0.4947 & 0.4865 & 0.0025 & 0.0158 & 0.0006 & 0.0134 & 0.001 & 0 & 0.013 & 0.0123 & 0.0009 & 0.0026 & 0.0224 & 0.0039 \\
\hline o & 10 & 0.0072 & 0.5835 & 0.0025 & 0.0003 & 0.0129 & 0.0009 & 0.0004 & 0.0299 & 0.013 & 0 & 0.0006 & 0.0001 & 0.4036 & 0.0066 & 0.0002 \\
\hline o & 11 & 0.0008 & 0.0016 & 0.4325 & 0.0108 & 0.0091 & 0.0011 & 0.0195 & 0.0004 & 0.0123 & 0.0006 & 0 & 0.0307 & 0.0001 & 0.1886 & 0.3161 \\
\hline o & 12 & 0.0019 & 0.0002 & 0.0044 & 0.4997 & 0.0007 & 0.0106 & 0.0146 & 0.0006 & 0.0009 & 0.0001 & 0.0307 & 0 & 0.0001 & 0.017 & 0.4638 \\
\hline $\mathrm{Cu}$ & 13 & 0.0147 & 0.0161 & 0.0003 & 0.0002 & 0.0015 & 0.0026 & 0.0001 & 0.4157 & 0.0026 & 0.4036 & 0.0001 & 0.0001 & 0 & 0.001 & 0.0001 \\
\hline $\mathrm{Cu}$ & 14 & 0.0105 & 0.0154 & 0.0523 & 0.0124 & 0.1604 & 0.0114 & 0.0131 & 0.0065 & 0.0224 & 0.0066 & 0.1886 & 0.017 & 0.001 & 0 & 0.0354 \\
\hline $\mathrm{Cu}$ & 15 & 0.0003 & 0.0003 & 0.0112 & 0.0165 & 0.001 & 0.0024 & 0.006 & 0.0002 & 0.0039 & 0.0002 & 0.3161 & 0.4638 & 0.0001 & 0.0354 & 0 \\
\hline
\end{tabular}

348-C

\begin{tabular}{|c|c|c|c|c|c|c|c|c|c|c|c|c|c|c|c|c|}
\hline & & Al & Al & Al & Al & o & o & o & o & o & o & o & o & $\mathrm{Cu}$ & $\mathrm{Cu}$ & $\mathrm{Cu}$ \\
\hline & & 1 & 2 & 3 & 4 & 5 & 6 & 7 & 8 & 9 & 10 & 11 & 12 & 13 & 14 & 15 \\
\hline Al & 1 & 0 & 0.0087 & 0.02 & 0.0185 & 0.401 & 0.0035 & 0.2471 & 0.5556 & 0.003 & 0.0086 & 0.003 & 0.3369 & 0.0164 & 0.0155 & 0.0048 \\
\hline Al & 2 & 0.0087 & 0 & 0.0024 & 0.0263 & 0.0044 & 0.0025 & 0.2886 & 0.01 & 0.5266 & 0.5935 & 0.0008 & 0.0036 & 0.0194 & 0.0007 & 0.0002 \\
\hline Al & 3 & 0.02 & 0.0024 & 0 & 0.0243 & 0.0068 & 0.4716 & 0.2815 & 0.0025 & 0.0032 & 0.0017 & 0.5601 & 0.2695 & 0.0003 & 0.0124 & 0.0233 \\
\hline Al & 4 & 0.0185 & 0.0263 & 0.0243 & 0 & 0.4198 & 0.4609 & 0.1067 & 0.0021 & 0.514 & 0.0024 & 0.0026 & 0.0024 & 0.0005 & 0.0193 & 0.0007 \\
\hline o & 5 & 0.401 & 0.0044 & 0.0068 & 0.4198 & 0 & 0.0195 & 0.0168 & 0.0115 & 0.0141 & 0.001 & 0.0034 & 0.0126 & 0.0016 & 0.1262 & 0.012 \\
\hline o & 6 & 0.0035 & 0.0025 & 0.4716 & 0.4609 & 0.0195 & 0 & 0.0186 & 0.0008 & 0.0131 & 0.0007 & 0.0122 & 0.0104 & 0.0005 & 0.0694 & 0.004 \\
\hline o & 7 & 0.2471 & 0.2886 & 0.2815 & 0.1067 & 0.0168 & 0.0186 & 0 & 0.0088 & 0.0208 & 0.0112 & 0.0085 & 0.0128 & 0.0019 & 0.0033 & 0.0019 \\
\hline o & 8 & 0.5556 & 0.01 & 0.0025 & 0.0021 & 0.0115 & 0.0008 & 0.0088 & 0 & 0.0012 & 0.0291 & 0.0006 & 0.0079 & 0.4246 & 0.0033 & 0.0008 \\
\hline o & 9 & 0.003 & 0.5266 & 0.0032 & 0.514 & 0.0141 & 0.0131 & 0.0208 & 0.0012 & 0 & 0.0155 & 0.0008 & 0.0006 & 0.0024 & 0.0049 & 0.0004 \\
\hline o & 10 & 0.0086 & 0.5935 & 0.0017 & 0.0024 & 0.001 & 0.0007 & 0.0112 & 0.0291 & 0.0155 & 0 & 0.0003 & 0.0005 & 0.4013 & 0.0006 & 0.0002 \\
\hline o & 11 & 0.003 & 0.0008 & 0.5601 & 0.0026 & 0.0034 & 0.0122 & 0.0085 & 0.0006 & 0.0008 & 0.0003 & o & 0.0375 & 0.0002 & 0.0114 & 0.4216 \\
\hline o & 12 & 0.3369 & 0.0036 & 0.2695 & 0.0024 & 0.0126 & 0.0104 & 0.0128 & 0.0079 & 0.0006 & 0.0005 & 0.0375 & 0 & 0.0018 & 0.0603 & 0.2171 \\
\hline $\mathrm{Cu}$ & 13 & 0.0164 & 0.0194 & 0.0003 & 0.0005 & 0.0016 & 0.0005 & 0.0019 & 0.4246 & 0.0024 & 0.4013 & 0.0002 & 0.0018 & 0 & 0.0006 & 0.0002 \\
\hline $\mathrm{Cu}$ & 14 & 0.0155 & 0.0007 & 0.0124 & 0.0193 & 0.1262 & 0.0694 & 0.0033 & 0.0033 & 0.0049 & 0.0006 & 0.0114 & 0.0603 & 0.0006 & o & 0.023 \\
\hline $\mathrm{Cu}$ & 15 & 0.0048 & 0.0002 & 0.0233 & 0.0007 & 0.012 & 0.004 & 0.0019 & 0.0008 & 0.0004 & 0.0002 & 0.4216 & 0.2171 & 0.0002 & 0.023 & 0 \\
\hline
\end{tabular}

348-D

\begin{tabular}{|c|c|c|c|c|c|c|c|c|c|c|c|c|c|c|c|c|}
\hline & & Al & Al & Al & Al & o & o & o & o & o & o & o & o & $\mathrm{Cu}$ & $\mathrm{Cu}$ & $\mathrm{Cu}$ \\
\hline & & 1 & 2 & 3 & 4 & 5 & 6 & 7 & 8 & 9 & 10 & 11 & 12 & 13 & 14 & 15 \\
\hline Al & 1 & 0 & 0.0107 & 0.0277 & 0.0196 & 0.5263 & 0.0099 & 0.0023 & 0.3922 & 0.4875 & 0.0057 & 0.0052 & 0.0008 & 0.0074 & 0.0019 & 0.000 \\
\hline Al & 2 & 0.0107 & 0 & 0.0184 & 0.002 & 0.0037 & 0.0039 & 0.0006 & 0.3687 & 0.0017 & 0.3731 & 0.5283 & 0.3015 & 0.017 & 0.0198 & 0.00 \\
\hline Al & 3 & 0.0277 & 0.0184 & 0 & 0.0155 & 0.4856 & 0.4594 & 0.0024 & 0.0045 & 0.004 & 0.0018 & 0.4742 & 0.0015 & 0.0007 & 0.0014 & 0.00 \\
\hline Al & 4 & 0.0196 & 0.002 & 0.0155 & 0 & 0.0035 & 0.4993 & 0.4485 & 0.0022 & 0.4926 & 0.0015 & 0.0024 & 0.0061 & 0.0004 & 0.0602 & 0.01 \\
\hline o & 5 & 0.5263 & 0.0037 & 0.4856 & 0.0035 & 0 & 0.02 & 0.0005 & 0.0148 & 0.0197 & 0.0006 & 0.0178 & 0.0005 & 0.0045 & 0.0012 & 0. \\
\hline o & 6 & 0.0099 & 0.0039 & 0.4594 & 0.4993 & 0.02 & 0 & 0.0129 & 0.0018 & 0.0232 & 0.0006 & 0.0145 & 0.0014 & 0.0004 & 0.0105 & 0.0 \\
\hline o & 7 & 0.0023 & 0.0006 & 0.0024 & 0.4485 & 0.0005 & 0.0129 & 0 & 0.0003 & 0.0119 & 0.005 & 0.0003 & 0.0151 & 0.0009 & 0.2161 & 0.1 \\
\hline o & 8 & 0.3922 & 0.3687 & 0.0045 & 0.0022 & 0.0148 & 0.0018 & 0.0003 & 0 & 0.0129 & 0.0116 & 0.0147 & 0.0081 & 0.1047 & 0.0078 & \\
\hline o & 9 & 0.4875 & 0.0017 & 0.004 & 0.4926 & 0.0197 & 0.0232 & 0.0119 & 0.0129 & 0 & 0.0006 & 0.0012 & 0.0014 & 0.0016 & 0.0115 & 0.0 \\
\hline o & 10 & 0.0057 & 0.3731 & 0.0018 & 0.0015 & 0.0006 & 0.0006 & 0.005 & 0.0116 & 0.0006 & 0 & 0.0095 & 0.9488 & 0.1569 & 0.0504 & 0.001 \\
\hline o & 11 & 0.0052 & 0.5283 & 0.4742 & 0.0024 & 0.0178 & 0.0145 & 0.0003 & 0.0147 & 0.0012 & 0.0095 & 0 & 0.0113 & 0.0041 & 0.0034 & 0.0 \\
\hline o & 12 & 0.0008 & 0.3015 & 0.0015 & 0.0061 & 0.0005 & 0.0014 & 0.0151 & 0.0081 & 0.0014 & 0.9488 & 0.0113 & 0 & 0.0267 & 0.3022 & 0.0 \\
\hline $\mathrm{Cu}$ & 13 & 0.0074 & 0.017 & 0.0007 & 0.0004 & 0.0045 & 0.0004 & 0.0009 & 0.1047 & 0.0016 & 0.1569 & 0.0041 & 0.0267 & 0 & 0.018 & 0.00 \\
\hline $\mathrm{Cu}$ & 14 & 0.0019 & 0.0198 & 0.0014 & 0.0602 & 0.0012 & 0.0105 & 0.2161 & 0.0078 & 0.0115 & 0.0504 & 0.0034 & 0.3022 & 0.018 & 0 & 0.05 \\
\hline & 15 & .0006 & 0.0003 & 0.0003 & 0.0139 & 0.0002 & 0.0021 & 0.1751 & 0.0001 & 0.0045 & 0.001 & 0.0001 & .004 & 0.0004 & .0561 & \\
\hline
\end{tabular}


Table S3 Coordinates of stable isomers.

046-A

\begin{tabular}{|c|c|c|c|}
\hline atom & $x$ & $y$ & $z$ \\
\hline $\mathrm{Al}$ & -0.30969 & 1.1100 & 1.1167 \\
\hline $\mathrm{Al}$ & 0.11538 & 1.3717 & -1.3466 \\
\hline $\mathrm{Al}$ & -0.31971 & -1.0811 & -1.0128 \\
\hline $\mathrm{O}$ & -0.048190 & -0.10438 & 2.2535 \\
\hline $\mathrm{O}$ & 0.51706 & 2.2587 & 0.080790 \\
\hline $\mathrm{O}$ & 0.50635 & -0.082240 & -2.1943 \\
\hline $\mathrm{O}$ & -0.057700 & -2.1840 & 0.23240 \\
\hline $\mathrm{O}$ & 0.14949 & -3.0160 & 3.1680 \\
\hline $\mathrm{O}$ & -1.1341 & 0.40523 & -0.34623 \\
\hline
\end{tabular}

046-B

\begin{tabular}{|c|c|c|c|}
\hline atom & $x$ & $y$ & $z$ \\
\hline $\mathrm{Al}$ & -0.78883 & 1.2060 & 0.90745 \\
\hline $\mathrm{Al}$ & 1.0431 & 0.83624 & -1.1091 \\
\hline $\mathrm{Al}$ & -1.2420 & -1.0567 & -0.58459 \\
\hline $\mathrm{O}$ & 0.24116 & 0.24191 & 1.9078 \\
\hline $\mathrm{O}$ & 2.0568 & -0.25287 & -0.27015 \\
\hline $\mathrm{O}$ & -0.13207 & -2.0673 & 0.23110 \\
\hline $\mathrm{O}$ & -2.0579 & 0.23689 & 0.17601 \\
\hline $\mathrm{O}$ & 0.13065 & 2.0510 & -0.32784 \\
\hline $\mathrm{O}$ & -0.22632 & -0.24146 & -1.8732 \\
\hline
\end{tabular}

046-C

\begin{tabular}{|c|c|c|c|}
\hline atom & $x$ & $y$ & $z$ \\
\hline $\mathrm{Al}$ & 4.4977 & 0.59155 & 0.083060 \\
\hline $\mathrm{Al}$ & 0.96215 & 0.39979 & 1.9787 \\
\hline $\mathrm{Al}$ & 3.1633 & -0.83635 & 1.6803 \\
\hline $\mathrm{O}$ & 0.75740 & 1.6370 & 0.80174 \\
\hline
\end{tabular}




\begin{tabular}{|c|c|c|c|}
\hline $\mathrm{O}$ & 4.8698 & -0.55969 & 1.3100 \\
\hline $\mathrm{O}$ & 3.8666 & 2.1599 & -0.36130 \\
\hline $\mathrm{O}$ & 1.4159 & -1.3243 & 1.9865 \\
\hline $\mathrm{O}$ & 2.5054 & 0.44106 & 2.8309 \\
\hline $\mathrm{O}$ & 2.7820 & -0.068170 & -0.00070000 \\
\hline
\end{tabular}

047-A

\begin{tabular}{|c|c|c|c|}
\hline atom & $x$ & $y$ & $z$ \\
\hline $\mathrm{Al}$ & -0.71818 & -1.5303 & 0.25154 \\
\hline $\mathrm{Al}$ & -0.72739 & 1.5199 & 0.29077 \\
\hline $\mathrm{Al}$ & -2.6512 & -0.0044400 & -0.24380 \\
\hline $\mathrm{O}$ & -1.3202 & -0.017270 & 1.0672 \\
\hline $\mathrm{O}$ & -2.2249 & -1.6306 & -0.64223 \\
\hline $\mathrm{O}$ & 0.94177 & 1.4515 & 0.094700 \\
\hline $\mathrm{O}$ & -2.2347 & 1.6339 & -0.60026 \\
\hline $\mathrm{O}$ & 0.95050 & -1.4468 & 0.057380 \\
\hline $\mathrm{O}$ & 3.5731 & 0.020410 & -0.72759 \\
\hline $\mathrm{O}$ & 3.6382 & 0.0029800 & 0.63906 \\
\hline
\end{tabular}

047-B

\begin{tabular}{|c|c|c|c|}
\hline atom & $x$ & $y$ & $z$ \\
\hline $\mathrm{Al}$ & 3.1815 & 2.2457 & -0.029100 \\
\hline $\mathrm{Al}$ & 3.5382 & 2.4963 & 2.4670 \\
\hline $\mathrm{Al}$ & 0.99490 & 2.2412 & 2.8509 \\
\hline $\mathrm{O}$ & 1.8041 & 1.7919 & -0.93852 \\
\hline $\mathrm{O}$ & 2.4771 & 2.2678 & 3.7907 \\
\hline $\mathrm{O}$ & 4.5150 & 2.1113 & 1.0800 \\
\hline $\mathrm{O}$ & -0.27442 & 3.6161 & 2.2808 \\
\hline $\mathrm{O}$ & -0.45992 & 3.4840 & 0.97338 \\
\hline $\mathrm{O}$ & 0.38981 & 1.0299 & 1.7752 \\
\hline $\mathrm{O}$ & 2.1483 & 2.9342 & 1.3346 \\
\hline
\end{tabular}


047-C

\begin{tabular}{|c|c|c|c|}
\hline Atom & $x$ & $y$ & $z$ \\
\hline $\mathrm{Al}$ & -1.76194 & 0.37308 & 0.83397 \\
\hline $\mathrm{Al}$ & 0.34013 & -0.01775 & 1.98173 \\
\hline $\mathrm{Al}$ & 0.88100 & -0.73321 & -0.59202 \\
\hline $\mathrm{O}$ & -1.14825 & -1.96139 & -0.07965 \\
\hline $\mathrm{O}$ & -0.81316 & 1.28653 & 1.95300 \\
\hline $\mathrm{O}$ & 0.35622 & -2.37760 & -0.82138 \\
\hline $\mathrm{O}$ & 1.63201 & -0.16846 & 0.85196 \\
\hline $\mathrm{O}$ & -3.05393 & -1.74854 & -0.13174 \\
\hline $\mathrm{O}$ & -3.36989 & -0.55192 & 0.34559 \\
\hline $\mathrm{O}$ & -0.96971 & -1.24727 & 1.57669 \\
\hline
\end{tabular}

347-A

\begin{tabular}{|c|c|c|c|}
\hline Atom & $x$ & $y$ & $z$ \\
\hline $\mathrm{Al}$ & -1.3982 & -0.49937 & 1.5117 \\
\hline $\mathrm{Al}$ & -1.2532 & 2.2665 & 0.65269 \\
\hline $\mathrm{Al}$ & 0.66911 & 1.0796 & -1.1357 \\
\hline $\mathrm{Cu}$ & -1.2452 & -2.7162 & 0.074690 \\
\hline $\mathrm{Cu}$ & 1.1613 & -1.0526 & 0.49935 \\
\hline $\mathrm{Cu}$ & 3.8789 & 0.15864 & -0.81170 \\
\hline $\mathrm{O}$ & -2.5650 & 1.9942 & -0.44757 \\
\hline $\mathrm{O}$ & 0.28260 & 2.4209 & -0.12270 \\
\hline $\mathrm{O}$ & 2.0390 & 0.071310 & -0.78512 \\
\hline $\mathrm{O}$ & -2.3599 & -0.86344 & 0.045340 \\
\hline $\mathrm{O}$ & -0.68368 & 0.37482 & -1.9568 \\
\hline $\mathrm{O}$ & -0.22747 & -1.7774 & 1.5530 \\
\hline
\end{tabular}


347-B

\begin{tabular}{|c|c|c|c|}
\hline atom & $x$ & $y$ & $z$ \\
\hline $\mathrm{Al}$ & -1.6912 & -0.46583 & 1.1344 \\
\hline $\mathrm{Al}$ & -1.2058 & 2.3930 & 0.87791 \\
\hline $\mathrm{Al}$ & 0.88389 & 1.3954 & -0.78282 \\
\hline $\mathrm{Cu}$ & -0.63281 & -3.4974 & 1.9806 \\
\hline $\mathrm{Cu}$ & 0.99815 & -1.0051 & 0.63162 \\
\hline $\mathrm{Cu}$ & 3.9179 & 0.13470 & -0.29885 \\
\hline $\mathrm{O}$ & -2.3169 & 2.4709 & -0.44642 \\
\hline $\mathrm{O}$ & 0.45242 & 2.5655 & 0.40621 \\
\hline $\mathrm{O}$ & 2.0842 & 0.19429 & -0.38360 \\
\hline $\mathrm{O}$ & -2.4132 & -0.42656 & -0.42976 \\
\hline $\mathrm{O}$ & -0.33951 & 0.97865 & -1.9202 \\
\hline $\mathrm{O}$ & -0.54299 & -1.7273 & 1.4988 \\
\hline
\end{tabular}

347-C

\begin{tabular}{|c|c|c|c|}
\hline atom & $x$ & $y$ & $z$ \\
\hline $\mathrm{Al}$ & 1.2419 & -1.3942 & 0.94351 \\
\hline $\mathrm{Al}$ & 1.3389 & 2.1953 & -0.21348 \\
\hline $\mathrm{Al}$ & -0.90559 & 0.82863 & 0.94679 \\
\hline $\mathrm{Cu}$ & -1.4228 & -1.5609 & -0.12726 \\
\hline $\mathrm{Cu}$ & 0.90185 & -0.90839 & -1.5918 \\
\hline $\mathrm{Cu}$ & -4.1787 & 0.37590 & 0.38667 \\
\hline $\mathrm{O}$ & 0.35050 & -2.2729 & -0.27441 \\
\hline $\mathrm{O}$ & 0.32254 & -0.12754 & 1.6880 \\
\hline $\mathrm{O}$ & 2.9387 & -1.0160 & 0.81909 \\
\hline $\mathrm{O}$ & -2.3782 & 0.024410 & 0.52683 \\
\hline $\mathrm{O}$ & 1.8478 & 0.76801 & -1.1909 \\
\hline $\mathrm{O}$ & -0.29989 & 2.2896 & 0.24251 \\
\hline
\end{tabular}


347-D

\begin{tabular}{|c|c|c|c|}
\hline atom & $x$ & $y$ & $z$ \\
\hline $\mathrm{Al}$ & -1.7361 & -0.38421 & 1.1816 \\
\hline $\mathrm{Al}$ & -1.1596 & 2.4389 & 0.85944 \\
\hline $\mathrm{Al}$ & 0.82095 & 1.2914 & -0.94742 \\
\hline $\mathrm{Cu}$ & -1.5654 & -2.4093 & -0.53822 \\
\hline $\mathrm{Cu}$ & 0.86027 & -1.0528 & 0.51426 \\
\hline $\mathrm{Cu}$ & 2.2912 & 1.4405 & 1.2671 \\
\hline $\mathrm{O}$ & -2.2914 & 2.5498 & -0.43559 \\
\hline $\mathrm{O}$ & 0.51104 & 2.4827 & 0.34259 \\
\hline $\mathrm{O}$ & 2.0180 & 0.22815 & -0.28963 \\
\hline $\mathrm{O}$ & -2.4964 & -0.35218 & -0.43096 \\
\hline $\mathrm{O}$ & -0.47608 & 0.99011 & -2.0297 \\
\hline $\mathrm{O}$ & -0.75530 & -1.8105 & 1.1802 \\
\hline
\end{tabular}

347-E

\begin{tabular}{|c|c|c|c|}
\hline atom & $x$ & $y$ & $z$ \\
\hline $\mathrm{Al}$ & -1.5776 & -1.3693 & 0.35340 \\
\hline $\mathrm{Al}$ & 3.0814 & -0.60901 & -0.31549 \\
\hline $\mathrm{Al}$ & 0.41806 & 0.19766 & 1.5293 \\
\hline $\mathrm{Cu}$ & -1.2872 & 1.2962 & -0.21558 \\
\hline $\mathrm{Cu}$ & 2.0865 & 1.8893 & 0.050810 \\
\hline $\mathrm{Cu}$ & -3.9063 & -0.22164 & -1.7677 \\
\hline $\mathrm{O}$ & 1.6510 & -0.93225 & 0.90463 \\
\hline $\mathrm{O}$ & -0.98081 & -0.74843 & 1.8494 \\
\hline $\mathrm{O}$ & 3.6500 & 0.94979 & -0.43100 \\
\hline $\mathrm{O}$ & 0.29634 & 1.7588 & 0.81418 \\
\hline $\mathrm{O}$ & -0.50369 & -2.5624 & -0.30784 \\
\hline $\mathrm{O}$ & -2.4846 & -0.24381 & -0.59398 \\
\hline
\end{tabular}


348-A

\begin{tabular}{|c|c|c|c|}
\hline atom & $x$ & $y$ & $z$ \\
\hline $\mathrm{Al}$ & 0.082160 & -0.51883 & 0.56422 \\
\hline $\mathrm{Al}$ & -2.2909 & 1.5065 & 0.82383 \\
\hline $\mathrm{Al}$ & 2.2335 & 0.85150 & -0.33038 \\
\hline $\mathrm{Cu}$ & -0.65736 & -0.80836 & -1.9852 \\
\hline $\mathrm{Cu}$ & 3.4525 & -1.3839 & 0.36451 \\
\hline $\mathrm{Cu}$ & -2.8729 & -1.3390 & 0.44663 \\
\hline $\mathrm{O}$ & -1.6081 & 2.7850 & -0.10285 \\
\hline $\mathrm{O}$ & -0.68787 & 0.89297 & 1.3274 \\
\hline $\mathrm{O}$ & -3.5121 & 0.31032 & 0.78183 \\
\hline $\mathrm{O}$ & 1.8105 & -0.61725 & 0.89426 \\
\hline $\mathrm{O}$ & -1.1444 & -1.6273 & -0.084540 \\
\hline $\mathrm{O}$ & 0.45370 & 0.54679 & -0.97302 \\
\hline $\mathrm{O}$ & 1.7236 & 2.4381 & 0.15783 \\
\hline
\end{tabular}

348-B

\begin{tabular}{|c|c|c|c|}
\hline atom & $x$ & $y$ & $z$ \\
\hline $\mathrm{Al}$ & -1.8866 & 1.1126 & -0.28155 \\
\hline $\mathrm{Al}$ & 0.88484 & 1.5969 & -1.0309 \\
\hline $\mathrm{Al}$ & 1.8250 & -1.2031 & -0.91824 \\
\hline $\mathrm{Cu}$ & 3.5182 & 0.80324 & 0.36779 \\
\hline $\mathrm{Cu}$ & -3.8531 & -1.1359 & -0.47285 \\
\hline $\mathrm{Cu}$ & 0.61216 & 0.86531 & 1.4721 \\
\hline $\mathrm{O}$ & 3.3472 & -0.90507 & -0.14616 \\
\hline $\mathrm{O}$ & 0.60401 & -2.2387 & -0.30713 \\
\hline $\mathrm{O}$ & -2.5366 & -2.3202 & -0.14518 \\
\hline $\mathrm{O}$ & -0.97133 & -0.060310 & 0.72908 \\
\hline $\mathrm{O}$ & 1.9636 & 1.8200 & 0.36951 \\
\hline $\mathrm{O}$ & 1.2597 & 0.14463 & -1.8501 \\
\hline
\end{tabular}




\begin{tabular}{|l|l|l|l|}
\hline $\mathrm{O}$ & -3.4786 & 0.62807 & -0.59980 \\
\hline
\end{tabular}

348-C

\begin{tabular}{|c|c|c|c|}
\hline atom & $x$ & $y$ & $z$ \\
\hline $\mathrm{Al}$ & -1.1649 & 0.74714 & -1.3234 \\
\hline $\mathrm{Al}$ & 1.2318 & -1.8209 & -0.92214 \\
\hline $\mathrm{Al}$ & -1.3189 & -1.7105 & -0.22118 \\
\hline $\mathrm{Cu}$ & -0.78614 & 3.2414 & -0.57855 \\
\hline $\mathrm{Cu}$ & 3.0768 & -0.55935 & 0.98004 \\
\hline $\mathrm{Cu}$ & -2.6938 & 0.31980 & 1.1636 \\
\hline $\mathrm{O}$ & -2.3281 & -0.54760 & -1.0631 \\
\hline $\mathrm{O}$ & 0.33366 & -0.24140 & -0.81803 \\
\hline $\mathrm{O}$ & -1.0400 & 2.2847 & -2.1182 \\
\hline $\mathrm{O}$ & -1.0001 & 1.6142 & 0.41910 \\
\hline $\mathrm{O}$ & -0.15345 & -2.8367 & -0.81981 \\
\hline $\mathrm{O}$ & 2.8363 & -1.7121 & -0.40053 \\
\hline $\mathrm{O}$ & -1.0691 & -0.94381 & 1.3674 \\
\hline
\end{tabular}

348-D

\begin{tabular}{|c|c|c|c|}
\hline atom & $x$ & $y$ & $z$ \\
\hline $\mathrm{Al}$ & -2.0104 & -1.1991 & 0.13902 \\
\hline $\mathrm{Al}$ & -2.4321 & 1.0901 & -0.72344 \\
\hline $\mathrm{Al}$ & 0.038240 & 2.2797 & -0.052230 \\
\hline $\mathrm{Cu}$ & 2.2508 & 0.51863 & 0.64994 \\
\hline $\mathrm{Cu}$ & 0.71411 & -2.3125 & 0.49689 \\
\hline $\mathrm{Cu}$ & -0.14474 & -0.10933 & 1.8380 \\
\hline $\mathrm{O}$ & 0.81752 & 1.7136 & -1.4850 \\
\hline $\mathrm{O}$ & -1.6656 & 2.5343 & -0.31140 \\
\hline $\mathrm{O}$ & 1.6642 & -1.0335 & -0.63831 \\
\hline $\mathrm{O}$ & -0.98696 & -1.8400 & 1.3362 \\
\hline $\mathrm{O}$ & -3.3715 & -0.13193 & 0.046050 \\
\hline
\end{tabular}




\begin{tabular}{|c|c|c|c|}
\hline $\mathrm{O}$ & -1.1887 & -0.22582 & -1.2200 \\
\hline $\mathrm{O}$ & 0.70540 & 1.5450 & 1.3728 \\
\hline
\end{tabular}

